\author{
Alberto Preda ${ }^{1 *}$, Giorgio Fiore ${ }^{1 *}$, Carlo Gaspardone ${ }^{1}$, Silvana Di Maio ${ }^{1}$, Davide Romagnolo ${ }^{1}$, \\ Lorenzo Rampa', Luigia Brugliera², Andrea Tettamanti², Carlo Meloni ${ }^{2}$, Sandro lannaccone' ${ }^{2}$ \\ ${ }^{1}$ Clinical Cardiology Unit, IRCCS Ospedale San Raffaele, Milano, Italy \\ ${ }^{2}$ IRCCS Ospedale San Raffaele, Milano, Italy \\ *A. Preda and G. Fiore equally contributed to this work and should be considered as shared first authors
}

\title{
Study on the utility and efficacy of clinical and instrumental tests in the follow-up of COVID-19 patients
}

\section{Corresponding author:}

Giorgio Fiore, Clinical Cardiology Unit, IRCCS Ospedale San Raffaele, Milano, Italy, e-mail: gio.fio@hotmail.it
Medical Research Journal 2021; Volume 6, Number 3, 169-176 10.5603/MRJ.a2021.0037 Copyright $\odot 2021$ Via Medica ISSN 2451-2591 e-ISSN 2451-4101

\begin{abstract}
Introduction: The disease caused by the SARS-CoV-2 virus (COVID-19) frequently leads to serious complications and prolonged hospitalizations requiring effective care after discharge.

Aim of the study: Aim of this study was to identify feasible and cost-effective predictors of outcome among clinical characteristics, functional status, laboratory, echocardiographic and lung ultrasound data of COVID-19 patients.

Material and methods: Patients affected by COVID-19 who experienced a prolonged hospitalization due to a severe form of the disease and that have been discharged from the COVID-19 rehabilitation unit (RU) were prospectively enrolled between April $6^{\text {th }}$ and May $22^{\text {nd }}, 2020$. All the patients underwent a 6-minute walk test (6MWT) at the 30-day follow-up. Baseline characteristics, laboratory, functional exercise tests, echocardiographic and lung ultrasound (LUS) data collected between hospitalization, admission to RU, discharge from RU and follow-up were compared. Correlations with the predicted distance covered at the 6MWT (6MWD) were made.

Results: 40 patients met inclusion criteria and presented to follow-up (13 women [32.5\%] and 27 men [67.5\%]; mean age $66 \pm 10$ years). Among all variables analysed, only functional tests at discharge showed a remarkable correlation with the 6MWD. Significant improvement in lung ultrasound score (LUSS) was also observed however without correlation with 6MWD.

Conclusions: functional tests at discharge from RU identified patients with different 30-day outcomes that could deserve a stricter long-term follow-up. This may help in planning a personalized follow-up. The costs and effort were minimal. The severity of the acute phase did not significantly influence functional recovery. LUS was useful to identify subclinical lung damage and its evolution over time, however without clear functional correlation.
\end{abstract}

Key words: COVID-19, lung ultrasound, rehabilitation, SARS-CoV-2

Med Res J 2021; 6 (3): 169-176

\section{How these fit in:}

- Little is known about the long-term functional status of discharged COVID-19 patients that experienced a severe disease needing rehabilitation.

- Given the variability of clinical pictures led by COVID-19 and the continuous increase of patients' discharge, cost-free and easy to use, efficient tools for planning the follow-up and stratify prognosis are needed.
- In a selected population of COVID-19 patients with loss of functional autonomy that required a rehabilitation period, commonest functional tests showed to correlate well with $6 \mathrm{MWT}$ performance at 30 days from discharge.

- Lung ultrasound sonography is a safe, feasible and cost-effective method to monitor lung damage over time. 


\section{Introduction}

The coronavirus disease of 2019 (COVID-19) is a viral illness caused by the severe acute respiratory syndrome coronavirus 2 (SARS-CoV-2), with large variability of clinical pictures [1]. Considering the high potential severity burden of long-term complications [2] and the need for prolonged hospitalizations [3], the establishment of rehabilitative departments dedicated to most compromised patients was deemed necessary [4-6]. In this context, an amount of literature discussing patient in-hospital management was published [7]. Nevertheless, remain a lack of evidence about adequate follow-up planning [8] because insight about the long-term clinical outcome and functional status is still missing $[9,10]$. Considering the enormous number of outpatients to manage and the limited resources, the research should be oriented towards the identification of harmless, feasible and cost-effective tools that easily provide information on individual risk and help to select patients who need closer follow-up. Other than physiological characteristics, many laboratory and instrumental tests are used as risk predictors of mortality during hospitalization and at discharge [11], however without clear evidence of long-term benefit. Chest computed tomography (CT) is a critical tool for the diagnosis and inpatient management, with higher sensitivity than a swab sample [12] capable to identify lung alterations even at 60 days of follow-up [13] in asymptomatic individuals [14]. Despite this, it may not be a feasible method for the routine follow-up of discharged COVID-19 patients because of radiation exposure, costs, availability, and logistic issues. On the other hand, lung ultrasound sonography (LUS) appears to be a useful, rapid, harmless and low-cost alternative to CT, with similar sensitivity in COVID-19 $[15,16]$ and capable to identify subclinical residual lung damage in patients with severe COVID-19 that met discharge criteria [17]. Exercise tests and functional scales are quantitative validated tools to assess and train the functional reserve in several rehabilitation units, being useful to stratify patients risk according to their fitness. Among them, the 6 Minute Walk Test (6MWT) is a submaximal exercise test used to assess aerobic capacity and endurance, providing a measure of functional status and outcome in patients affected by different lung and cardiac diseases [18-20]. So far, the only test that found application in the context of the COVID-19 pandemic was the 6MWT, which demonstrated effectiveness in assessing oxygenation reserve in non-hypoxic patients at rest and help in looking for discharge preparedness $[27,28]$. Moreover, in the largest cohort study with the longest follow-up available today, 6MWT at 6 months was decreased proportionally to the severity of illness [29]. This study aimed to identify feasible and cost-effective predictors of outcome among clinical characteristics, laboratory and functional tests, echocardiographic and LUS data in a selected high-risk cohort of COVID-19 patients who needed a rehabilitative recovery. This might help to identify frailer patients deserving a stricter follow-up strategy and to avoid the routine use, even during the acute phase of the disease, of useless laboratory and diagnostic tests with a huge waste of resources. The authors used the 6MWT as a functional endpoint, being this an effective, and easy to perform predictor of prognosis, largely validated in several lung and cardiac diseases $[18,19]$.

\section{Material and methods}

\section{Study population}

We prospectively enrolled consecutive COVID-19 patients who were hospitalized during the acute phase of the disease in the Emergency Room (ER), Intensive Care Units (ICU), Respiratory High Dependency Care Units (RHDCU) or Infectious Diseases units of the San Raffaele Hospital, that were subsequently admitted to a dedicated Rehabilitation Unit (RU) and underwent a 30-day post-discharge follow-up between April 6 and July 2, 2020. Criteria to admit COVID-19 patients in the RU were: positive swab for SARS-CoV-2, stable $\mathrm{SatO}_{2}$ and respiratory rate, no need for respiratory assistance or no more than $2 \mathrm{l} / \mathrm{min} \mathrm{O}_{2}$, absence of fever, presence of areas of dependence at the FIM evaluation (FIM score < 100) [4]. For the present study, exclusion criteria were the presence of acute cardio-pulmonary or inflammatory conditions, not COVID-19 related (i.e., acute heart failure, COPD exacerbation, pulmonary embolism), concurrent condition influencing functional tests (i.e., traumatic injury), patient refusal or loss at the 30-day follow-up and death. All patients gave their informed consent, and the study was approved by the local Ethics Committee.

\section{Laboratory tests}

Based on the reported haematological findings of COVID-19 patients, the following inflammatory indexes were considered: white blood cells (WBCs) and subtypes counts, C-reactive protein (CRP), serum ferritin, and D-dimer [30]. The N-terminal pro-brain natriuretic peptide (NT-proBNP) level was also assessed, which is an independent risk factor for in-hospital death in patients with severe COVID-19 [31]. Laboratory data were assessed during the hospitalization (acute phase of the disease) before admission to the $\mathrm{RU}$ and were part of the COVID-BioB Study [32]. 


\section{Transthoracic echocardiography}

Wide spectrum cardio-pulmonary involvement is common in COVID-19 [33]. Transthoracic echocardiography at discharge from RU and follow-up was performed by a trained cardiologist blinded to the patient's clinical characteristics, aimed at identifying signs of right ventricle (RV) dysfunction and/or pressure overload: The authors evaluated RV longitudinal systolic function by tricuspid annular plane systolic excursion (TAPSE) and lateral tricuspid annular tissue Doppler imaging (S' TDI). Pulmonary artery systolic pressure (PASP) was estimated by a sum of tricuspid regurgitation jet gradient and estimated right atrial pressure derived from analysis of the inferior vena cava (IVC) dimensions and response to inspiration. Images were obtained with GE VividS60 (GE-Healthcare, Chicago, Illinois) equipped with a $3 \mathrm{Sc}$-RS sector transducer probe.

\section{Lung US (LUS)}

Lung US at admission and discharge from the RU and at 30-day follow-up was performed with the patient in a sitting position by a single trained operator blinded to the patient's clinical characteristics. Findings were classified according to a validated quantitative LUS Score (LUSS) [34]. Three areas per hemithorax were identified (anterior, lateral, and posterior) by using the anterior and posterior axillary lines as anatomical landmarks. Each area was then divided into two, superior and inferior [35]. Therefore, a total of 12 thoracic areas was considered. Each area was given a score from 0 to 3 according to the following criteria: 0 , normal aeration; 1 , more than $2 \mathrm{~B}$-lines occupying $50 \%$ of the pleura or less; 2 , more than 2 B-lines occupying greater than $50 \%$ of the pleura; and 3, tissue-like pattern. Therefore, the total LUSS ranged from 0 to 36 . Lung US images were obtained with a Prosound $\alpha 6$ system (Hitachi Aloka Medical Systems, Tokyo, Japan) equipped with a UST9123 convex transducer.

\section{Functional evaluation}

Functional status and independence in daily living activities were assessed at the admission of the RU, discharge from RU and 30-day follow-up with tests used in Rehabilitation Units and selected for this kind of patient [6]: 6MWT [36], TUG [21] and 30CST [23]. Timed up and go test (TUG) [21] is a measure of functional mobility validated in patients affected by chronic obstructive pulmonary disease and pulmonary arterial hypertension [22]. It evaluates the time a patient takes to rise from a chair, walk three meters, turn around, walk back to the chair and sit down. The Thirty-second chair-stand test (30CST) evaluates leg strength and endurance in older adults assessing the number of stands that a person can complete in 30 seconds [23, 24]. Activities of daily living (ADL) is commonly assessed using the Functional Independence Measure (FIM) scale, an 18-item measurement tool that explores the level of a patient's disability and indicates how much assistance is required for the individual to perform daily living activities [25]. By adding the points for each item ( 1 = total assist and $7=$ complete independence), the level of independence ranges from 18 (lowest) to 126 (highest) [26]. The 6MWT can be expressed as absolute distance and percentage of the predicted 6-minutes walking distance (6MWD). A validated reference equation was used for the prediction of the total distance walked during six minutes for healthy adults [38]. The distance covered over a time of 6 minutes is used to compare changes in performance capacity.

\section{Statistical analysis}

For each continuous variable, normal distribution by the Shapiro-Wilk test was verified. Normally distributed variables were described as mean \pm standard deviation while non-normally distributed ones were described as medians (interquartile range). The comparisons between groups were performed using t-tests or Wilcoxon sum-rank tests, as appropriate.

The categorical variables were described as frequencies (percentages) and compared by Chi-squared tests. The relationship between variables was exhibited by using the Spearman Rank Correlation coefficient. A two-sided $P$ value $<0.05$ was required for statistical significance. Data were analysed with $\mathrm{R}$ software version 3.6.2 (R Foundation for Statistical Computing, Vienna, Austria).

\section{Results}

Forty patients (13 women [32.5\%] and 27 men [67.5\%]; mean age $66 \pm 10$ years) met inclusion criteria and presented to the follow-up (Fig. 1). Baseline demographic and clinical characteristics of the population are reported in Table 1. Eighteen patients (45\%) required ventilatory support during the acute disease phase. Comorbidities were present in $57.5 \%$ of patients, with hypertension being the most prevalent one (50\%). 36 patients (90\%) received at least 1 antimicrobial or immunosuppressant COVID-19 treatment, with hydroxychloroquine being the most frequent one (77.5\%). Patients baseline characteristics, treatment during the acute phase (hospitalization), laboratory findings, functional tests, echocardiographic data and LUSS at discharge from RU were correlated with the distance covered at the 6MWT at the 30-day follow-up. 


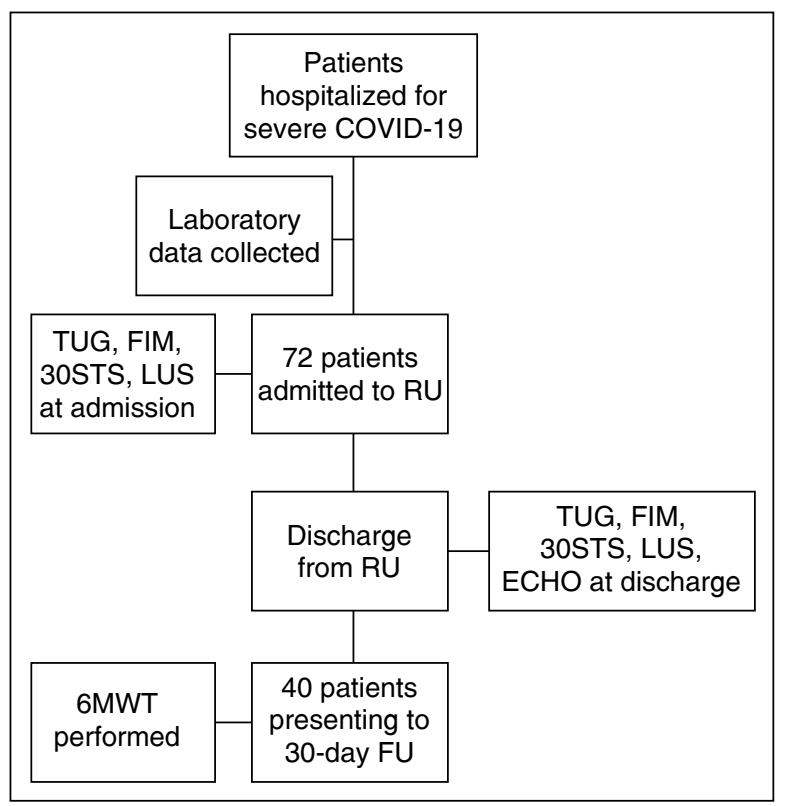

Figure 1. Flow diagram of study structure.

30 STS - 30 seconds sit-to-stand; TUG - time up-andgo; FIM - functional independence measure; LUS - lung ultrasound; ECHO - transthoracic echocardiography; $\mathrm{RU}$ - rehabilitation unit; FU — follow-up

(Tab. 3). Considering comorbidities, diabetes was associated with the worst performance at 6MWT ( $p=0.02$ ). Patients treated with lopinavir-ritonavir combination or IL-6 inhibitors performed significantly better at the 6MWT at follow-up. No difference was observed between patients treated with other drugs. Eighteen patients (45\%) scored a 6MWT lower than the predicted for age, weight and height. Interestingly, a patient needing ventilatory support during the acute phase did not perform worse at the 30-day 6MWT ( $p=0.399)$. Regarding functional evaluation, this analysis showed that all TUG, FIM and 30CTS at discharge from RU significantly correlated to the 6MWD at follow-up (Tab. 3, Fig. 2) and markedly improved throughout the observation period from discharge from RU to the 30-day follow-up (Tab. 2). No correlation was found between laboratory tests, echocardiographic parameters at discharge from $\mathrm{RU}$ and follow-up and the distance covered at the 6MWT.

Similarly, no correlation was observed between LUSS at discharge from RU, LUSS at follow-up and 6MWT. About Lung US findings, median LUSS at follow-up was significantly lower when compared with LUSS at discharge from RU (8.0 vs. 3.0, p < 0.001; Tab. $2)$. The same trend was observed both in patients that needed ventilatory support during the acute phase of the disease (8.0 vs. $4.0 p<0.001)$ and those who did not $(2.5$ vs. $0.0 p=0.05)$ and it was significantly higher in the first group at discharge from RU and follow-up
Table 1. Baseline demographic and clinical characteristics of the population

\begin{tabular}{|c|c|}
\hline Variable & \\
\hline No. Patients, n & 40 \\
\hline Age, mean \pm DS & $66 \pm 10$ \\
\hline Female, n (\%) & $13 / 40(32.5 \%)$ \\
\hline Body mass index, $\mathrm{Kg} / \mathrm{m} 2 \pm \mathrm{DS}$ & $25.7 \pm 4.9$ \\
\hline Current smoking status, n (\%) & $3 / 40(7.5 \%)$ \\
\hline Need for endotracheal intubation (n\%) & $18 / 40(45 \%)$ \\
\hline 6MWT lower than the predicted & $18 / 40(45 \%)$ \\
\hline Comorbidities, n (\%) & \\
\hline Hypertension & $20 / 40(50 \%)$ \\
\hline Diabetes & $9 / 40(22.5 \%)$ \\
\hline Chronic obstructive lung disease & $2 / 40(5 \%)$ \\
\hline Coronary artery disease & $5 / 40(12.5 \%)$ \\
\hline Chronic kidney disease & $5 / 40(12.5 \%)$ \\
\hline Chronic therapy with ACE-i or ARBs & $16 / 40(40 \%)$ \\
\hline COVID-19 treatment, n (\%) & \\
\hline Hydroxychloroquine & $31 / 40(77.5 \%)$ \\
\hline Antibiotics (Azithromycin) & $20 / 40(50.5 \%)$ \\
\hline Antiviral drugs (Lopinavir - Ritonavir) & $26 / 40(65 \%)$ \\
\hline IL-1 inhibitor (Anakinra) & $4 / 40(10 \%)$ \\
\hline $\begin{array}{l}\text { IL-6 inhibitors (Tocilizumab, } \\
\text { Sarilumab) }\end{array}$ & $7 / 70(10 \%)$ \\
\hline $\begin{array}{l}\text { Laboratory findings at discharge, } \\
\text { median (IQR) }\end{array}$ & \\
\hline White blood cell count, $\times 109 / \mathrm{L}$ & $5.7(2.7)$ \\
\hline Lymphocyte count, ×109/L & $1.7(1.0)$ \\
\hline C-reactive protein, $\mathrm{mg} / \mathrm{L}$ & $5.95(7.7)$ \\
\hline Serum ferritin, $\mu \mathrm{g} / \mathrm{L}$ & $401.50(650)$ \\
\hline D-dimer, $\mu \mathrm{g} / \mathrm{L}$ & $0.42(0.305)$ \\
\hline NT-pro-BNP, pg/mL & $167(461)$ \\
\hline
\end{tabular}

ACE-I - Angiotensin-converting enzyme inhibitors; ARBs - Angiotensin II receptor blockers; COVID-19 - coronavirus disease 2019; $\mathrm{IL}$ - Interleuchine

(Fig. 3). A positive trend from admission to RU (median 8, IQR 9) to discharge from RU (median 8, IQR 8, $p=0.0413$ ) was also found.

\section{Discussion}

\section{Summary}

Our study led to the following results: 1) Among a variety of clinical, laboratory and instrumental data in a selected population of Covid-19 patients with loss of 
Table 2. LUSS, echocardiography and functional test findings of patients at discharge from COVID-19 Rehabilitation Unit and after 30 days

\begin{tabular}{lccc}
\hline Variable & Discharge & Follow-up & P-value \\
\hline Lung ultrasound, median (IQR) & & & $<(5.75)$ \\
LUS score, points & $8(8)$ & & 0.001 \\
Transthoracic echocardiography of the RV, mean \pm DS & & $27.2 \pm 8.0$ & 0.328 \\
Estimated PASP, mmHg & $28.9 \pm 4.8$ & $21.5 \pm 4.6$ & 0.092 \\
TAPSE, mm & $23.3 \pm 4.6$ & $14.8 \pm 4.4$ & 0.008 \\
Lateral tricuspid S' TDI, cm/s & $17.5 \pm 4.5$ & & 0.0036 \\
Functional Tests, median (IQR) & & $12(6)$ & 0.00038 \\
30 STS test, No. of repetitions & $10.5(5)$ & $9(5)$ & $<0.00001$ \\
TUG test, seconds & $11(5)$ & $125(7.75)$ & $(14.5)$ \\
FIM scale, points & 110 & \\
\hline
\end{tabular}

Bold for statistically significant values at $\mathrm{p}<0.05$. IQR — interquartile range; LUS — lung ultrasound; RV — right ventricle; PASP — pulmonary arterial systolic pressure; TAPSE — tricuspid annular plane systolic excursion; TDI — tissue doppler imaging 30 STS, 30 seconds sit-to-stand; TUG — time up-and-go; FIM — functional independence measure

functional autonomy that required a rehabilitation period only functional evaluation through TUG, FIM and 30CTS showed to correlate well with the prognosis evaluated by $6 \mathrm{MWT}$ at 30 days from discharge; 2) the severity of the acute phase of the disease did not influence functional recovery; 3) LUSS significantly improved from admission to RU to discharge form RU and 30day follow-up, however without functional correlation at the $6 \mathrm{MWT}$.

\section{Follow-up and long-term outcome of severe COVID-19 patients}

Nowadays there is a poorness of shared strategies for the follow-up of patients that suffered from severe COVID-19 requiring strict monitoring. There is also little knowledge about functional long-term outcomes, the presence of residual lung damage and its clinical correlation. In this pilot single-centre study, even limited by low sample size and a short-term follow-up, it was looked for a correlation between a variety of clinical and functional tests, evaluated before discharge from the rehabilitation unit of the hospital, and the functional outcome using the 6MWT, a simple, economic, reproducible and largely validated prognostic predictive test. In a population of patients that presented with clinical indications for functional rehabilitation (FIM < 100) shortly after the onset of the disease and immediately after the acute phase of the infection, this analysis showed that functional tests performed at discharge (TUG, 30-CTS, FIM) positively correlated with the distance covered at the 6MWT at the follow-up, helping to identify frailer patients needing a stricter and personalized follow-up strategy after discharge. On the other hand, no laboratory test before discharge (white blood cell and subtype counts, C-reactive protein, serum ferritin, D-dimer and
$\mathrm{N}$-terminal pro-brain natriuretic peptide) nor echocardiographic parameters (right ventricular function, PASP) correlates with 6MWD at the 30-day follow-up.

\section{Ventilatory support and functional recovery}

An interesting aspect that emerged from this analysis is that patients that needed ventilatory support during the acute phase of the disease, experiencing a more severe pulmonary involvement also confirmed by higher LUSS, performed worse at the 30-CTS test before discharge, but no difference emerged at the 30-day follow-up. Similarly, there was no difference between the two groups and the 6MWT performance at the 30-day follow-up. This result may signify that the severity of the acute phase, once discharge criteria are met, does not influence the functional long-term outcome. This data is in line with a recent study that highlighted the incongruity between the severity of respiratory disease and cognitive outcome [38]. The authors hope that this notable acknowledgement would be confirmed in larger population observational studies.

\section{Lung ultrasound in the follow-up of discharged patients}

Lung ultrasound is a central tool for the diagnosis and management of hospitalised COVID-19 patients, owning several advantages in terms of safety, costs, comfort and availability in respect to radiological instruments [6, 26, 27]. In the present study, the 30-day LUSS was significantly lower compared to LUSS at discharge. Thus, there was a clear reduction of parenchymal involvement within 30 days both in the group of patients that needed mechanical ventilation and those who did not. Furthermore, patients experiencing a more severe 
Table 3. Correlations between variables in the study and the percentage of the predicted value of 6 minutes walking test

\begin{tabular}{|c|c|}
\hline Qualitative Variables & P-value \\
\hline Need for endotracheal intubation & 0.399 \\
\hline Current smoking status & 0.082 \\
\hline Chronic therapy with RAAS Inhibitors & 0.98 \\
\hline \multicolumn{2}{|l|}{ Comorbidities } \\
\hline Hypertension & 0.37 \\
\hline Diabetes & 0.02 \\
\hline Chronic obstructive lung disease & 0.91 \\
\hline Coronary artery disease & 0.09 \\
\hline \multicolumn{2}{|l|}{ COVID-19 treatment } \\
\hline Hydroxychloroquine & 0.36 \\
\hline Antibiotics (Azithromycin) & 0.31 \\
\hline Antiviral drugs (Lopinavir-Ritonavir) & 0.059 \\
\hline IL-1 inhibitor (Anakinra) & 0.82 \\
\hline IL-6 inhibitors (Tocilizumab, Sarilumab) & 0.027 \\
\hline Quantitative Variables & r coefficient \\
\hline Body mass index & 0.4 \\
\hline \multicolumn{2}{|l|}{ Laboratory findings at discharge } \\
\hline White blood cell count & -0.2 \\
\hline Lymphocyte & -0.2 \\
\hline C-reactive protein & -0.14 \\
\hline D-dimer & -0.04 \\
\hline Serum ferritin & 0.004 \\
\hline NT-pro-BNP & -0.21 \\
\hline \multicolumn{2}{|l|}{ Lung Ultrasound } \\
\hline LUSS at discharge & -0.04 \\
\hline LUSS at follow-up & -0.14 \\
\hline \multicolumn{2}{|l|}{ Echocardiography of the $\mathrm{RV}$ at discharge } \\
\hline Estimated PASP & 0.52 \\
\hline TAPSE & 0.24 \\
\hline Lateral tricuspid S' TDI & 0.04 \\
\hline \multicolumn{2}{|c|}{ Echocardiography of the RV at Follow-up } \\
\hline Estimated PASP & -0.16 \\
\hline TAPSE & 0.22 \\
\hline Lateral tricuspid S' TDI & 0.37 \\
\hline \multicolumn{2}{|l|}{ Functional Tests at discharge } \\
\hline 30 STS test & $\begin{array}{l}0.58(95 \mathrm{Cl} \\
0.25-0.79 \\
\mathrm{p}=0.002)\end{array}$ \\
\hline TUG test & $\begin{array}{l}-0.66(95 \mathrm{Cl} \\
0.42-0.82 ; \\
p<0.001)\end{array}$ \\
\hline FIM scale & $\begin{array}{l}0.57(95 \mathrm{Cl} \\
0.30-0.75 \\
\mathrm{p}<0.001)\end{array}$ \\
\hline \multicolumn{2}{|l|}{ Functional Tests at follow-up } \\
\hline 30 STS test & 0.65 \\
\hline TUG test & -0.55 \\
\hline FIM scale & 0.48 \\
\hline
\end{tabular}

Discharge refers to discharge from the rehabilitation unit. Bold for statistically significant values at $p<0.05$. RAAS - Renin Angiotensin Aldosterone System; COVID-19 - coronavirus disease 2019; IL — Interleuchine; LUS - lung ultrasound; RV — right ventricle; PASP — pulmonary arterial systolic pressure; TAPSE — tricuspid annular plane systolic excursion; TDI - tissue doppler imaging; 30 STS -30 seconds sit-to-stand; TUG — time up-and-go; FIM — functional independence measure

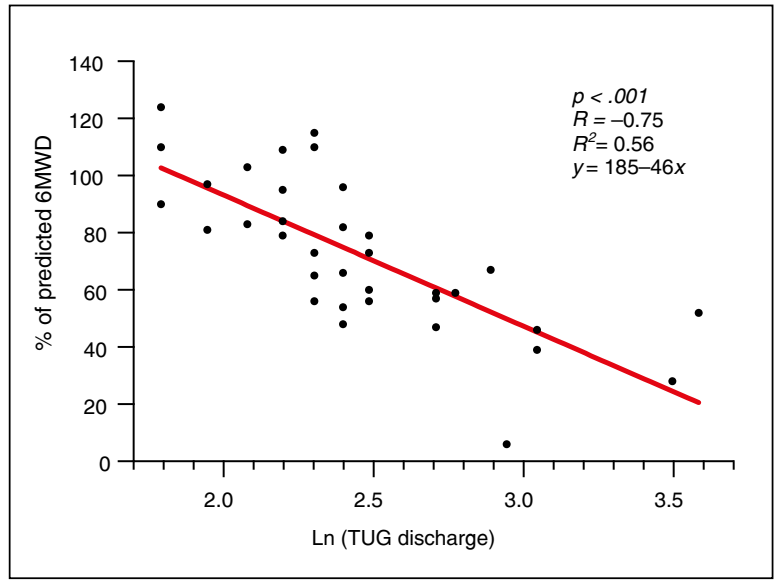

Figure 2. Correlation between TUG (values expressed as natural logarithm) and the distance covered at 6MWT

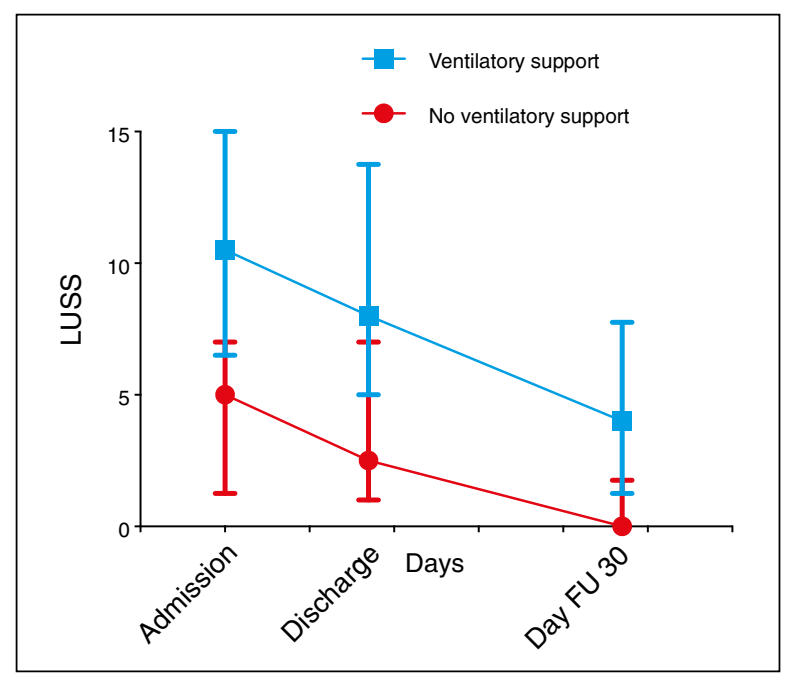

Figure 3. Median LUSS from admission to discharge and follow-up in patients needing and not needing ventilatory support during the acute phase of the disease. The median LUSS was higher in patients needing ventilatory support $(p<0.05)$

disease (needing ventilatory support) had a higher LUSS at the admission of the RU, discharge from RU and 30-day follow-up. However, no correlation was found between LUSS at discharge, LUSS at follow-up and 6MWT. This interesting result may be explained by the high sensitivity of the method, revealing residual lung involvement even without clinical and/or functional correlation.

\section{Implications for research and/or practice}

Most of the tests commonly used to predict outcome in COVID-19 during the acute phase failed in predicting 
the long-term outcome availed at the follow-up by the $6 \mathrm{MWT}$, a well-validated predictor of mortality. Therefore, new risk predictors to consider for customized follow-up planning aimed at the optimization of available resources may be needed. Routine use of instrumental or expensive tests (i.e., pro-BNP, echocardiography, contrast-enhanced CT, LUS etc.) in the absence of specific condition (i.e., heart failure, signs of pulmonary hypertension, pulmonary embolism) should be avoided, as they failed to correlate with the clinical and functional outcome of the patient and, as in the case of echocardiogram, may raise the risk of infection spread among healthcare professionals. On the contrary, all the functional tests that were considered (TUG, 30CTS, FIM) seemed to be the most useful and strongest predictors of outcome at 30-day.

\section{Conflict of interests: The authors declare no competing interests.}

\section{Funding: None.}

\section{References}

1. Huang $C$, Wang $Y, L i X$, et al. Clinical features of patients infected with 2019 novel coronavirus in Wuhan, China. Lancet. 2020; 395(10223): 497-506, doi: 10.1016/S0140-6736(20)30183-5, indexed in Pubmed: 31986264

2. Marshall M. The lasting misery of coronavirus long-haulers. Nature. 2020; 585(7825): 339-341, doi: 10.1038/d41586-020-02598-6, indexed in Pubmed: 32929257.

3. Rees EM, Nightingale ES, Jafari Y, et al. COVID-19 length of hospital stay: a systematic review and data synthesis. BMC Med. 2020; 18(1): 270, doi: 10.1186/s12916-020-01726-3, indexed in Pubmed: 32878619

4. Iannaccone S, Castellazzi P, Tettamanti A, et al. Role of rehabilitation department for adult individuals with COVID-19: The experience of the San Raffaele Hospital of Milan. Arch Phys Med Rehabil. 2020; 101(9): 1656-1661, doi: 10.1016/j.apmr.2020.05.015, indexed in Pubmed: 32505489 .

5. Wade DT Rehabilitation after COVID-19: an evidence-based approach Clin Med (Lond). 2020; 20(4): 359-365, doi: 10.7861/clinmed.2020-0353, indexed in Pubmed: 32518105.

6. Brugliera L Spina A Castellazzi P et al. Rehabilitation of COVID-19 patients. J Rehabil Med. 2020; 52(4): jrm00046, doi: 10.2340/165019772678, indexed in Pubmed: 32286674

7. Negrini F, de Sire A, Andrenelli E, et al. International Multiprofessional Steering Committee of Cochrane Rehabilitation REH-COVER action. Rehabilitation and COVID-19: a rapid living systematic review 2020 by Cochrane Rehabilitation Field. Update as of October 31st, 2020 Eur J Phys Rehabil Med. 2021: 57(1): 166-170, doi: 10.23736/S19739087.20.06723-4, indexed in Pubmed: 33263249.

8. Balachandar V, Mahalaxmi I, Subramaniam M, et al. Follow-up studies in COVID-19 recovered patients - is it mandatory? Sci Total Environ 2020; 729: 139021, doi: 10.1016/j.scitotenv.2020.139021, indexed in Pubmed: 32360909.

9. Taboada M, Cariñena A, Moreno E, et al. Post-COVID-19 functional status six-months after hospitalization. J Infect. 2021; 82(4): e31-e33, doi: 10.1016/j.jinf.2020.12.022, indexed in Pubmed: 33373650

10. Cortinovis M, Perico N, Remuzzi G. Long-term follow-up of recovered patients with COVID-19. Lancet. 2021; 397(10270): 173-175, doi: 10.1016/S0140-6736(21)00039-8, indexed in Pubmed: 33428868.

11. Du RH, Liang $L R$, Yang $C Q$, et al. Predictors of mortality for patients with COVID-19 pneumonia caused by SARS-CoV-2: a prospective cohort study. Eur Respir J. 2020; 55(5), doi: 10.1183/13993003.00524-2020, indexed in Pubmed: 32269088.
12. Ai T, Yang Z, Hou H, et al. Correlation of chest CT and RT-PCR testing for Coronavirus Disease 2019 (COVID-19) in China: A report of 1014 cases. Radiology. 2020; 296(2): E32-E40, doi: 10.1148/radiol.2020200642. indexed in Pubmed: 32101510

13. Lanza E, Profili M, Bolengo I, et al. COVID-19 lung alterations still evident at 60-day follow-up chest CT in asymptomatic patients despite negative rRT-PCR testing. 2020, doi: 10.21203/rs.3.rs-28202/v1.

14. Tabata S, Imai K Kawano S, et al Clinical characteristics of COVID-19 in 104 people with SARS-CoV-2 infection on the Diamond Princess cruise ship: a retrospective analysis. Lancet Infect Dis. 2020; 20(9): 1043-1050, doi: 10.1016/S1473-3099(20)30482-5, indexed in Pubmed: 32539988

15. Peng QY, Wang XT, Zhang LN, et al. Chinese Critical Care Ultrasound Study Group (CCUSG). Findings of lung ultrasonography of novel corona virus pneumonia during the 2019-2020 epidemic. Intensive Care Med. 2020; 46(5): 849-850, doi: 10.1007/s00134-020-05996-6, indexed in Pubmed: 32166346

16. Soldati $G$, Smargiassi $A$, Inchingolo $R$, et al. Is there a role for lung ultrasound during the COVID-19 pandemic? J Ultrasound Med. 2020; 39(7): 1459-1462, doi: 10.1002/jum.15284, indexed in Pubmed: 32198775.

17. Gaspardone C, Meloni C, Preda A, et al. Lung ultrasound in COVID-19 a role beyond the acute phase? J Ultrasound Med. 2021; 40(3): 503-511, doi: 10.1002/jum.15425, indexed in Pubmed: 32770687.

18. Giannitsi S, Bougiakli M, Bechlioulis A, et al. 6-minute walking test: a useful tool in the management of heart failure patients. Ther Adv Cardiovasc Dis. 2019; 13: 1753944719870084, doi: 10.1177/1753944719870084, indexed in Pubmed: 31441375.

19. Dajczman E, Wardini R, Kasymjanova G, et al. Six minute walk distance is a predictor of survival in patients with chronic obstructive pulmonary disease undergoing pulmonary rehabilitation. Can Respir J. 2015; 22(4): 225-229, doi: 10.1155/2015/280187, indexed in Pubmed: 26252533

20. Rasekaba T, Lee AL, Naughton MT, et al. The six-minute walk test: a useful metric for the cardiopulmonary patient. Intern Med J. 2009 39(8): 495-501, doi: 10.1111/j.1445-5994.2008.01880.x, indexed in Pubmed: 19732197.

21. Podsiadlo D, Richardson S. The timed "Up \& Go": a test of basic functional mobility for frail elderly persons. J Am Geriatr Soc. 1991; 39(2): 142-148, doi: 10.1111/j.1532-5415.1991.tb01616.x, indexed in Pubmed: 1991946.

22. Mesquita R, Wilke $\mathrm{S}, \mathrm{Smid} \mathrm{DE}$, et al. Measurement properties of the Timed Up \& Go test in patients with COPD. Chron Respir Dis. 2016 13(4): 344-352, doi: 10.1177/1479972316647178, indexed in Pubmed: 27165963

23. Vaidya T, Chambellan A, de Bisschop C. Sit-to-stand tests for COPD: A literature review. Respir Med. 2017; 128: 70-77, doi: 10.1016/j. rmed.2017.05.003, indexed in Pubmed: 28610673.

24. Kidd D, Stewart G, Baldry J, et al. The Functional Independence Measure: a comparative validity and reliability study. Disabil Rehabil. 1995: 17(1): 10-14, doi: 10.3109/09638289509166622, indexed in Pubmed: 7858276

25. Gillen G. Overview of Cognitive and Perceptual Rehabilitation. Cognitive and Perceptual Rehabilitation. 2009: 1-31, doi: 10.1016/b978-0-32304621-3.10001-4.

26. Sasanuma N, Takahashi K, Tanaka T, et al. Functional independence measure analysis in patients undergoing phase I of cardiac rehabilitation. European Heart Journal. 2013: 34(suppl 1): P5801-P5801, doi: 10.1093/eurheartj/eht310.p5801.

27. Pandit R, Vaity C, Mulakavalupil B, et al. Unmasking Hypoxia in COVID 19 - Six Minute Walk Test. J Assoc Physicians India. 2020; 68(9): 50-51, indexed in Pubmed: 32798345 .

28. Fuglebjerg $\mathrm{NJ}$, Jensen TO, Hoyer $\mathrm{N}$, et al. Silent hypoxia in patients with SARS CoV-2 infection before hospital discharge. Int J Infect Dis. 2020; 99: 100-101, doi: 10.1016/j.ijid.2020.07.014, indexed in Pubmed: 32663601.

29. Huang C, Huang L, Wang Y, et al. 6-month consequences of COVID-19 in patients discharged from hospital: a cohort study. Lancet. 2021; 397(10270): 220-232, doi: 10.1016/S0140-6736(20)32656-8, indexed in Pubmed: 33428867.

30. Terpos E, Ntanasis-Stathopoulos I, Elalamy I, et al. Hematological findings and complications of COVID-19. Am J Hematol. 2020; 95(7): 834-847, doi: 10.1002/ajh.25829, indexed in Pubmed: 32282949.

31. Guo T, Fan Y, Chen M, et al. Cardiovascular implications of fatal outcomes of patients with coronavirus disease 2019 (COVID-19). JAMA Cardiol. 2020; 5(7): 811-818, doi: 10.1001/jamacardio.2020.1017, indexed in Pubmed: 32219356.

32. Zangrillo A, Beretta L, Scandroglio AM, et al. COVID-BioB Study Group , COVID-BioB Study Group. Characteristics, treatment, outcomes and cause of death of invasively ventilated patients with COVID-19 ARDS 
in Milan, Italy. Crit Care Resusc. 2020 [Epub ahead of print]; 22(3) indexed in Pubmed: 32353223.

33. Szekely $Y$, Lichter $Y$, Topilsky $Y$, et al. Spectrum of cardiac manifestations in COVID-19: A systematic echocardiographic study. Circulation. 2020; 142(4): 342-353, doi: 10.1161/CIRCULATIONAHA.120.047971, indexed in Pubmed: 32469253

34. Mongodi S, Bouhemad B, Orlando A, et al. Modified lung ultrasound score for assessing and monitoring pulmonary aeration. Ultraschal Med. 2017; 38(5): 530-537, doi: 10.1055/s-0042-120260, indexed in Pubmed: 28291991.

35. Bouhemad B, Mongodi S, Via G, et al. Ultrasound for "lung monitoring" of ventilated patients. Anesthesiology. 2015; 122(2): 437-447, doi: 10.1097/ALN.0000000000000558, indexed in Pubmed: 25501898.
36. ATS Committee on Proficiency Standards for Clinical Pulmonary Function Laboratories. ATS statement: guidelines for the six-minute walk test. Am J Respir Crit Care Med. 2002; 166(1): 111-117, doi: 10.1164/ajrccm.166.1.at1102, indexed in Pubmed: 12091180.

37. Enright PL, Sherrill DL. Reference equations for the six-minute walk in healthy adults. Am J Respir Crit Care Med. 1998; 158(5 Pt 1): 1384-1387, doi: 10.1164/ajrccm.158.5.9710086, indexed in Pubmed: 9817683

38. Alemanno F, Houdayer E, Parma A, et al. COVID-19 cognitive deficits after respiratory assistance in the subacute phase: A COVID-rehabilitation unit experience. PLoS One. 2021; 16(2): e0246590, doi: 10.1371/journal.pone.0246590, indexed in Pubmed: 33556127 